\title{
Liderazgo en Educación
}

\author{
Leadership in Education
}

\author{
Simón José Hernández Aponte \\ simher69@gmail.com \\ Universidad Militar Bolivariana de Venezuela
}

Recibido: 18-10-2016 / Revisado: 25-10-2016 / Aceptado: 16-11-2016 / Publicado: 10-04-2017

\section{RESUMEN}

Todo líder, cualesquiera que sean sus objetivos personales, debe ser útil a sus seguidores o no será líder, este es el jefe o dirigente de un grupo y la función que ejerce se denomina liderazgo, que se refiere por lo general al ejercicio de una autoridad que ha sido aceptada por el grupo y se supone que el líder de alguna manera es reconocido por sus capacidades, por su autoridad, o porque así lo determina el grupo al que pertenezca. El liderazgo no es exclusivo de unos cuantos, es tarea de todos. En cada individuo existe un gran potencial para motivar a otros y, de hecho, todos influimos en alguna medida en las personas con las que nos relacionamos. Todo educador es un líder, pues, además de su labor propiamente docente, lo cual implica un liderazgo intelectual, desempeña un papel trascendente en el desarrollo y la formación de actitudes y valores en los alumnos, hecho este que repercutirá en la vida de estos, y por consiguiente, en sus trabajos y en la sociedad donde se desenvuelvan. Es por ello, que el líder docente debe dominar sus competencias y a su vez, ser motivador del cambio en sus estudiantes a fin de generar las oportunidades para que estos logren alcanzar sus objetivos. En la educación, el liderazgo guarda una estrecha relación con su aceptación por parte de los integrantes del grupo. Tomando en cuenta la necesidad de estos, es el más adecuado en las relaciones interpersonales, escolares y educativas entre el docente y el alumno, por ende, es factor fundamental para lograr un proceso de enseñanzaaprendizaje satisfactorio. La búsqueda de la calidad, la excelencia y el liderazgo en la educación, supone un ejercicio permanente de corresponsabilidad, compartición y confluencia de voluntades en la perspectiva del interés grupal.

Palabras clave: Liderazgo, educación, enseñanza, estudiantes, autoridad

\begin{abstract}
Every leader, whatever his or her personal objectives, must be useful to his followers or he will not be a leader, this is the leader or leader of a group and the function he exercises is called leadership, which usually refers to the exercise of an authority which has been accepted by the group and it is assumed that the leader is somehow recognized by their abilities, by their authority, or because the group to which they belong determines it. Leadership is not exclusive to a few, it is everyone's job. In each individual there is great potential to motivate others and, in fact, we all influence to some extent the people with whom we relate. Every educator is a leader, because, in addition to his own teaching work, which implies an intellectual leadership, plays a transcendent role in the development and formation of attitudes and values in students, this fact that will affect their lives, and therefore, in their jobs and in the society where they operate. That is why the teacher leader must master their skills and, in turn, be a motivator of change in their students in order to generate opportunities for them to achieve their goals. In education, leadership is closely related to its acceptance by the members of the group. Taking into account the need for these, is the most appropriate in interpersonal, school and educational relations between the teacher and the student, therefore, is a key factor to achieve a satisfactory teachinglearning process. The search for quality, excellence and leadership in education is a permanent exercise of coresponsibility, sharing and confluence of wills in the perspective of group interest.
\end{abstract}

Key words: Leadership, education, teaching, students, authority 


\section{INTRODUCCIÓN}

El presente artículo sobre el liderazgo en educación busca enriquecer las vías que permitan alcanzar una educación de calidad. Es el resultado de un trabajo realizado en aulas donde se capacitan docentes para su participación en la educación superior de la Universidad "Alejandro Humboldt", Venezuela; donde se tomaron las opiniones y experiencias de alumnos y docentes, como bases para la iniciativa de escribir un artículo donde se plasme de manera sucinta la necesidad de formar educadores líderes a fin de poder influir en un grupo de personas que desean alcanzar una meta dentro del ámbito educativo y que a su vez, resalta la importancia del mejoramiento de los aprendizajes. El texto fue elaborado con un estilo simple y directo, dirigido fundamentalmente a los alumnos en área docente y profesores, así como estudiantes, especialistas e interesados en la formación o enseñanza. Este artículo nutre su contenido en bibliografía en liderazgo y educación. Su intención primera pasa por reflexionar respecto de las prácticas pedagógicas y la necesidad de que los docentes sean los líderes de las comunidades educativas. Los líderes escolares deben considerar que las limitaciones de pobreza, desigualdad social, presupuestos reducidos en educación, sueldos bajos de profesores, entre muchos otros factores, no determinan definitivamente el resultado de los aprendizajes en la escuela.

En vista de que el viejo paradigma educacional ha agotado su potencial para proporcionar una base teórica a los cambios en el área, se propone un nuevo paradigma capaz de sintetizar, operacionalizar e integrar algunas dimensiones que han ido apareciendo en los últimos diez años. El énfasis en el diseño de los cursos debe estar en perfiles abiertos y comprensivos con objetivos amplios y metas comprensivas. La relación con el instructor debe estar basada en el proceso de este cambio de paradigma, el cual implica una nueva cultura académico-instruccional en la que los tres centros en interacción son: estudiante, conocimiento y facilitador.

Todos los seres humanos tienen intrínsecamente habilidades que nutren a través de la educación formal e informal, que lo llevan a cumplir una función y de ella va a depender su figura principal más admirada, incluso idolatrada comenzando por el maestro quien permanece por mucho más tiempo en la vida diaria del individuo. Los alumnos tratarán de imitarlo en sus habilidades, con el propósito de poder surgir con las mismas condiciones o cualidades de su instructor.

\section{DISCUSIÓN Y RESULTADOS}

Primeramente, es básico definir Liderazgo, como la acción ejecutada por un Líder, entonces definiendo este último: como la persona a la que un grupo sigue aceptándola como su jefe. El término de líder se asocia al jefe o dirigente de un grupo y la función que ejerce es conocida como liderazgo. El líder es reconocido por sus capacidades, por su autoridad, o porque así lo determina el convenio del grupo. También podemos decir que liderazgo es una función muy especial y de gran responsabilidad para quienes la adquieran, toda vez que cada uno de los seres humanos la va asimilando paralelamente en su crecimiento personal bien sea: en la familia, en la vida estudiantil, en su grupo de amigos y compañeros de trabajo.

Pero un líder es un ser humano que, por error, lo califican perfecto, sin defectos, incapaz de errar. Porque un líder es un ser maduro, seguro, motivador, un gran comunicador, siempre tiene la respuesta y/o la solución, facilita y motiva. Es adaptable a las circunstancias, etc. Pero olvidan al humano que desarrolla habilidades para expresarse compartirlas y vivir en armonía y no un supremo. 
Existen varios tipos de autoridad, tanto moral, como civil, como militar, según el liderazgo que sea ejercido en base a principios morales o de valores, normas civiles o militares. En el ámbito de la educación existe liderazgo tanto en el sector docente como en el sector estudiantil, ambos quebrantados muchas veces por intereses propios más que por los de un grupo.

Un fuerte liderazgo educacional, en el que se dedique bastante tiempo a la coordinación, administración y simultáneamente se siga de cerca el proceso de enseñanza y socialización cotidiana del estudiante, es lo que necesita en este momento nuestra sociedad. En educación, se considera cliente al estudiante y su familia. Se entiende por satisfacción la capacidad de la institución para responder a las expectativas y necesidades de sus clientes. Una gestión de calidad significa conseguir los objetivos que uno se propone, facilitando los medios necesarios para ello.

El camino hacia la calidad es un camino difícil que exige un gran esfuerzo y colectivo que a veces compensa y otras produce verdadera frustración. Las instituciones educativas eficaces dan más importancia a la oferta; el instituto educativo ofrece unos modelos de eficacia que se fundamentan en su alto nivel de exigencia académico. Los modelos de gestión de la calidad dan una enorme importancia al liderazgo horizontal. Para que exista calidad es condición indispensable que la dirección ejerza un liderazgo claro y comprometido con los proyectos de mejora. La calidad de las instituciones educativas viene determinada por la visión, misión y valores de la satisfacción del cliente o educando.

El líder, que se mueve en el espacio de la autoridad para informar, se le exige integridad, fidelidad a principios y valores que comparte con sus seguidores; afronta el cambio, para lo cual transmite con emoción una visión de futuro, crea estrategias, fomenta el compromiso de los miembros del equipo y estimula para hacer frente a los obstáculos.
Los líderes más efectivos, dan más importancia a la participación de los subordinados en el control y la toma de decisiones. El estilo idóneo de dirección es el que es capaz de adecuarse a cada una de las situaciones que presentan los distintos subgrupos que conforman el equipo.

El liderazgo, en su funcionamiento parece sencillo, pero es un conjunto complejísimo de relaciones, sistemas y procesos que pocas personas dominan hoy en día en solitario. La principal preocupación del líder no es resolver el mismo los problemas, sino el comportamiento del grupo en el proceso de resolución de los mismos.

Nadie duda de la importancia del liderazgo para tres situaciones:

- Para facilitar el cambio y la innovación.

- Para proporcionar una visión de la organización.

- Para animar sus primeros pasos.

Al líder de las nuevas instituciones, no le queda más remedio que asumir el papel de facilitador que ayuda, con su visión cualificada y su misión compartida, a conseguir que el trabajo salga adelante con unos niveles de calidad verdaderamente competitivos.

El liderazgo no es una cualidad innata, es un proceso que se adquiere, que implica destreza y habilidades. Los líderes sobresalientes, poseen una visión personal del futuro de la institución, que suele ser compartida por una gran mayoría de colaboradores y que, en el fondo, impregnan todos los documentos institucionales de la organización como política y estrategia, los proyectos y los distintos planes de actuación de la vida cotidiana. La misión tiene una función simbólica y unificadora, sirve de marco, guía y criterio para valorar en cualquier momento la identidad y coherencia de la institución.

Hablar de educación, implica un tema muy amplio, en el que se deberían tocar muchos puntos, uno de ellos, y si se quiere uno de los de mayor interés es el de la educación 
superior. Las observaciones y criticas diarias tienen que ver con respecto a la falta de un cambio adecuado en nuestras universidades, un cambio en la enseñanza y formación de los futuros profesionales que se enfrentaran a los retos de una sociedad en constante cambio y evolución.

La innovación del trabajo académico que muchas veces se anhela y pretende de forma individual, (por una falta de liderazgo que permite la renovación del sistema docente, la transformación en la enseñanza y aún más a nivel superior), es un elemento que se debe considerar a la brevedad posible; muy por el contrario, a lo que actualmente se está aplicando en el sector Educación.

Hablando en el ámbito de la medicina, por ejemplo, se realizan numerosas prácticas ¿pero qué sucede al salir a la práctica rural? 0 aun peor, a la hospitalaria... que no es en nada parecida a todo ese mar de teorías que se aprendieron durante el estudio de la carrera. El cuerpo humano es un universo y a su vez cada paciente es diferente de otro, así se trate de la misma patología; nunca se nos explica cómo se debe entender primero al ser humano y tratar luego la enfermedad; a ponerse en los zapatos del otro y a jugárselas todas por todas, por el bienestar de quien lo requiere.

Así como esta carrera, todas ameritan una verdadera transformación, a lo que nos exige el mundo actual, para lo cual se hace necesario un liderazgo puro y auténtico, no el que suele predominar en nuestra sociedad, el del autoritarismo y el del más poderoso o fuerte.

La nueva concepción del liderazgo educativo rompe claramente con la relación jerárquica y vertical propia de culturas pasadas. El proceso educativo de enseñanza aprendizaje y el alumnado como protagonista fundamental del mismo, deben ser el núcleo de atención de los actos de liderazgo dentro de una escuela que trabaja por la calidad. Sin amor a los alumnos, no hay perfil de liderazgo educacional; El poder contenido como capacidad de mandar, dar órdenes y controlar desde una instancia personal, responde a la caricatura del pasado.

Un tipo de líder, es capaz de ayudar a tomar conciencia a los demás de sus posibilidades y capacidades, a liderar sus propias actividades, dentro de la organización pensando en su crecimiento y desarrollo profesional, (liderazgo transformacional). El líder transformacional, considera el trabajo en equipo como una estrategia importante que produce la sinergia necesaria, para conseguir mejores resultados a la organización. Los expertos en calidad total, piensan que es casi imposible conseguir resultados de calidad sin cuidar especialmente los procesos.

El proceso debe contar con un diseño previo de planificación en el que se tengan en cuenta claramente los ámbitos a los que se refiere, las necesidades y expectativas de los estudiantes a las que el proceso va a hacer frente y los costos del proceso medidos tanto en tiempo como en recursos disponibles.

Todo proceso debe incluir indicadores de progreso y calidad, que faciliten su seguimiento y la evaluación de resultados finales. En la gestión de la calidad total es fundamental la incentivación de las personas responsables, para que se impliquen en los procesos, provocando su creatividad y capacidad de innovación.

La mayor parte de los procesos que despliegan en un instituto de educación hacen referencia a cuatro ámbitos:

- Didácticos y académicos.

- Educativo de los valores.

- Administrativo y financiero.

- El ámbito de los servicios.

La evaluación de los resultados debe tener dos referentes que en la cultura de calidad total funcionan como criterios de evidencia, nos referimos a la satisfacción del estudiante y a la visión y misión de la institución.

El líder tiene importancia en la fase de la organización de los procesos y en la parte de 
mejora del proceso. Cuando se habla de objetivos de la educación, se destaca la preparación del ciudadano para integrarse en la sociedad como miembro productivo, es decir, como trabajador. El líder debe emplear todas sus energías en implicar a la mayor parte posible de colaboradores en la mayoría de las decisiones. El mejor lugar de aprendizaje y crecimiento profesional es el equipo de trabajo capaz de enriquecerse con los aportes de todos.

Es por ello, que es importante mostrar en todos los niveles de la educación al líder, sus características y tipos, para que así, ayude al docente a mejorar como ser humano, para guiarlo a ser un individuo mejor, que pueda compartir tanto individual como grupalmente dentro de una sociedad donde existen diferentes pensamientos, caracteres, niveles sociales etc. Que respete a sus semejantes desde todo punto de vista. Y pueda semejarse con facilidad, obteniendo sus propias metas y los objetivos grupales.

Un líder ideal es aquel que busca la más adecuada forma de comunicarse, respeta a sus semejantes, comparte y respeta el pensamiento del otro, tolerante y humilde, que involucra los grupos de trabajo, tomando en cuenta la voz y propuesta de ellos, sabe colocarse en el lugar del otro y facilita el trabajo, continuamente se actualiza y lo transmite. De esta forma es impartido sin restricciones en el futuro; podemos ser esos seres ideales que a través de la educación familiar y formal aseguraríamos la calidad de los futuros profesionales e individuos, aceptados en la sociedad, amplios y progresivos y no, como hasta ahora, han mal llamado líderes, a aquellos que solo buscaron su propio espacio y posición, al punto de crear profesionales $\mathrm{y} / \mathrm{o}$ individuos sin ética, mecanicistas, con intereses creados, con sed materialista y sin pensamiento progresista que ha llevado a esta sociedad a vivir en el egoísmo y la competencia desleal, cerrando el ciclo de la comunicación.
La base del cambio cultural se encuentra en los procesos de enseñanza aprendizaje; estos son verdaderos procesos de cambio de conducta, en los que intervienen elementos endógenos propios de la capacidades innatas del individuo, exógenos o inherentes a los diferentes agentes ambientales que permitirán, mediante los censores naturales, inducirlos bien, impactando los sentidos directamente a través del consciente o indirectamente, a través de los sistema de percepción en el sistema del subconsciente.

En cualquiera de los dos casos, la conformación de cada sistema, desde el punto de vista genético, impondrá diferentes niveles de captación del conocimiento, y este conocimiento puede ser adquirido, dependiendo del tipo de sistema impactando en la mente. En un conocimiento psicomotor, aquel percibido y aprendido demostrándose con las habilidades y destrezas de su sistema motor; cognoscitivo, que son aspectos teóricos del conocimiento e incluidos en la memoria, que se entrelaza con otros conocimientos $y$, por último, aquellos afectivos, que impactan los sentimientos y emociones.

El estudio y aplicación del liderazgo en la educación, podrá darnos las herramientas necesarias para conducir grupos de trabajo al lograr hacer más expedito su función y así satisfacer tanto las necesidades colectivas, como la principal necesidad personal. El liderazgo, además nos permitirá inducir entre los integrantes de una sociedad, la noción sobre el hecho de que las normas son también de ellos, producto de sus esfuerzos y que la comprensión de sus necesidades activó la forma de lograr el objeto educacional; por ello la formación con la cual debe intervenirse en las comunidades educativas. En todo trance, es necesario mantener un clima de unión entre los integrantes de una comunidad educativa, para que permita fluir el esfuerzo colectivo, y no solo la individualidad. En el diario ejecutar de acciones, colocar intereses particulares o grupales por encima de los intereses 
colectivos pervierte la intención del logro, que es el alcance del objeto educativo, claramente definido en los procesos de cualquier estructura social desarrollada.

Hay elementos que intervienen en el liderazgo educacional: un docente líder, es aquel con una serie de atributos que debe internalizar el proceso de formación educativa, con un comportamiento especial, para poder influir en las personas (alumnos) y motivarlas a cumplir con los objetivos que se desea alcanzar. Los atributos más importantes se relacionan tanto con los valores del buen ciudadano, como con los valores educacionales.

Atributos que debe poseer un líder:

a) Alta capacidad profesional.

b) Arraigados principios éticos.

c) Alto sentido de lealtad.

d) Honestidad a toda prueba.

e) Elevado nivel de entusiasmo.

f) Alta mística institucional.

El líder educador debe desarrollar de las siguientes características:

a) Tacto.

b) Seguridad en sí mismo.

c) Valor.

d) Sentido común.

e) Innovación.

f) Visión futuro.

Para ejercer un liderazgo propio, es necesario que el líder sea y se sienta un servidor, debe distinguirse por sus capacidades y habilidades, debe destacarse del grupo, diferenciarse de él y ser aceptado por él, no por su poder sino por su capacidad. Y es eso justamente lo que se ha perdido y lo que ha hecho que se sigan patrones dogmáticos y no se plantea el renovar ni el innovar.

La importancia de la relación bidireccional alumno -profesor, es otro aspecto que pasó a un segundo plano, ya no se respetan los puntos de vista ni las opiniones de cada cual, en muchas instituciones el educador es el Dios que todo lo sabe y que tiene todo el poder para reprobar el mayor número de estudiantes que sea posible, no se toma en cuenta el medio donde se desenvuelve ese alumno, las limitaciones que pueda tener y si no se conocen jamás se podrán ayudar a superar; es esa tal vez la esencia de la educación, él prepara integralmente el individuo para asumir los retos de una sociedad cada vez más moderna.

No es simplemente que el docente enfrente a su grupo con la autoridad de su cátedra, sin considerar a su auditorio, imponiendo sus puntos de vista, sin ni siquiera escuchar su alrededor; por el contrario, debe orientar su enseñanza según las expectativas de su grupo, haciéndolo parte de su planificación e involucrándolo más en este proceso que siempre debe considerarse dinámico. La capacitación diaria, las vivencias de nuestro día a día y la relación con los demás, con todas sus cualidades, inquietudes y limitaciones deben constituir el más importante ejemplo de educación, y en la medida que nos interesemos en el otro, al igual que en nosotros mismos y aprendemos a vivir con el otro y para el otro, seremos verdaderos líderes.

En educación el ejercicio del liderazgo guarda una estrecha relación con su aceptación por parte de los integrantes de un grupo. El profesor ha sido visto como una figura Autoritaria, más ese no es el liderazgo más deseable. Además del liderazgo democrático. En este último liderazgo la característica más relevante es que el líder considera los intereses, opiniones y la autoridad del grupo, convirtiéndose en un servidor, que busca enseñar y la vez aprender de los estudiantes. Este líder siempre piensa en el interés general del grupo. El docente debe ser ese líder que busque interpretar, orientar y conducir las inquietudes de sus estudiantes.

El docente debe ejercer un liderazgo efectivo entre el estudiantado, para que el proceso de enseñanza-aprendizaje pueda ser concretado. El docente debe ganarse el 
respeto de sus estudiantes, ya el sistema da la envergadura de autoridad en el aula, pero el título de líder debe ser un acuerdo entre él y sus estudiantes.

El líder docente, debe definirse como aquel que debe denominar sus funciones y tareas y con su competencia profesional e interés, mejorar la educación. Debe ser motivador de cambio en los estudiantes y crear oportunidades para ello. En el aula de clase se debe llegar a un acuerdo entre docente y estudiantes, para garantizar que ese liderazgo democrático contribuya a lograr el objetivo principal que es el aprendizaje.

La personalidad de quien ejerce el liderazgo educacional es un factor que también se aprende, se digiere, se internaliza y se analiza. Es por esto, que es necesario que el líder educador tenga cualidades y destrezas y domine ciertas habilidades que le permitan ejercer su liderazgo a conciencia plena de su responsabilidad. Por un lado, debe tener la pericia para definir los objetivos comunes del grupo en el que se desenvuelve. Debe atrapar la atención y el interés del grupo sobre el cual ejerce autoridad y estar a la vanguardia, debe ser portador de nuevas ideas sin tener miedo al cambio y a la innovación. Debe tener los conocimientos necesarios para captar los elementos psicológicos de los demás para poder entenderlos $\mathrm{y}$ tratarlos de forma individual y efectiva, según las características particulares de cada personalidad. Debe saber prever e intuir los problemas que podrían generar a futuro y en pos de esto, ejercer su habilidad en la toma de decisiones. Debe contagiar a los demás en la consecución de los logros, debe motivar y transmitir su energía a los demás de forma que alcancen sus objetivos colectivos. Debe tener la suficiente destreza, capacidad y habilidad para generar espacios de intercambio de forma que facilite la acción conjunta e individual. Estar dispuesto a aprender de los demás, estar abierto a los nuevos conocimientos, siempre en pos del perfeccionamiento y el aprendizaje, y no solo del colectivo sino personal. Es simplemente uno más que aprende y se perfecciona gracias a los otros, y es el intercambio con los demás que lo genera, sin embargo, dentro de esa estructura, es el líder que orienta el proceso en general.

En el área educativa, el líder debe tener conocimientos sobre la práctica docente, las teorías educativas, los modelos y técnicas de investigación de forma que en la práctica su liderazgo cumpla con el objetivo final de la educación relacionado a los conceptos de aprender-enseñar. Y finalmente para un liderazgo educacional efectivo debe tener la habilidad para aceptar a las personas como son y no pretender cambiarlas; debe tener la habilidad de confiar en los demás aun cuando el riesgo sea grande, es la confianza lo que da fortaleza a la debilidad del otro. Todas estas cualidades, capacidades y habilidades son inherentes al liderazgo educacional. La adquisición de esas habilidades y el desarrollo de esas destrezas, pueden ser efectivas en el liderazgo eficaz en la educación.

Sin embargo, se nos presenta ante nuestros ojos un nuevo problema y es que, con la globalización y la mediatización de la información, los canales para el aprendizaje escapan ya del aula escolar, y sometidos al bombardeo constante de información, los nuevos aprendices tienen ante sus fronteras una cantidad innumerable de mundos ya no solo físicos, sino también cibernéticos, los cuales muchas veces entran en pugna de valores y principios. Es por esto que hoy en día no basta con hacer efectivo el liderazgo, sino que además este liderazgo debe estar basado en la base de la corresponsabilidad, compartir y confluir de voluntades, de forma que se haga especial la generación de conocimientos deseada.

El docente es un líder, ya que posee las cualidades, habilidades y conocimientos necesarios para guiar el desarrollo a nivel personal, económico y social de sus alumnos. Un docente que carezca de empatía, humildad, 
valores, interés por los demás y ganas de dar lo mejor de sí, difícilmente será un líder aceptado por quienes deben seguirlo $y$, en definitiva, tampoco debería ser llamado docente.

\section{REFERENCIAS}

Bases del liderazgo en la educación; Líderes escolares, un tesoro para la educación. 1: $2006 . \quad$ Recuperado en: http://unesdoc.unesco.org/images/0014/0 01470/147055s.pdf.

El liderazgo educativo y su papel en la mejora: una revisión actual de sus posibilidades y limitaciones Psicoperspectivas. Individuo y Sociedad,. 9, (2) (2010). Recuperado en: http://www.psicoperspectivas.cl/index.ph $\mathrm{p} / \mathrm{psicoperspectivas/article/view/112/14}$ 0 .

Pérez Escarlín, Antonio (S/F). Liderazgo educativo. Recuperado en: https://antonioperezesclarin.com/2013/1 1/24/liderazgo-educativo/

Blog de gestión educativa. 10 cualidades del liderazgo educativo. Recuperado en: https://gestioneducativa.educaweb.com/10 -cualidades-del-liderazgo-educativo/ 